\title{
ACTIVIDADES DE OCIO Y CALIDAD DE VIDA DE LOS MAYORES EN LA COMUNIDAD DE MADRID ${ }^{1}$
}

\author{
Raúl Lardiés-Bosque \\ Departamento de Geografía y Ordenación del Territorio. Universidad de Zaragoza \\ rlardies@unizar.es \\ Fermina Rojo-Pérez \\ Instituto de Economía, Geografía y Demografía \\ Consejo Superior de Investigaciones Científicas (CSIC) \\ fermina.rojo@cchs.csic.es \\ Vicente Rodríguez-Rodríguez \\ Instituto de Economía, Geografía y Demografía \\ Consejo Superior de Investigaciones Científicas (CSIC) \\ vicente.rodriguez@cchs.csic.es \\ Gloria Fernández-Mayoralas \\ Instituto de Economía, Geografía y Demografía \\ Consejo Superior de Investigaciones Científicas (CSIC) \\ gloria.fernandezmayoralas@cchs.csic.es \\ María Eugenia Prieto-Flores \\ Departamento de Geografía. Universidad Nacional de Educación a Distancia \\ meprietof@geo.uned.es \\ Karim Ahmed-Mohamed \\ Instituto de Economía, Geografía y Demografía \\ Consejo Superior de Investigaciones Científicas (CSIC) \\ karim.ahmed@cchs.csic.es \\ José Manuel Rojo-Abuín \\ Unidad de Análisis Estadístico (UAE) \\ Consejo Superior de Investigaciones Científicas (CSIC) \\ josemanuel.rojo@cchs.csic.es
}

Fecha de recepción: febrero 2012.

Fecha de aceptación: enero 2013.

1 Esta investigación ha sido financiada con cargo a dos Proyectos de Investigación:

- Calidad de Vida de los mayores no institucionalizados en España. Una contribución al estudio de la Calidad de Vida desde una aproximación holística. (MCYT, Plan Nacional I+D+i 2000-2003, Ref. BSO200300401; I.P.: F. Rojo-Pérez).

- Evaluación de la Calidad de Vida de la población mayor en la Comunidad de Madrid. (Comunidad de Madrid, Plan Regional I+D+i, Ref. 06/HSE/0417/2004; I.P.: G. Fernández-Mayoralas).

Los autores dan las gracias a los mayores que aceptaron participar en la encuesta, por compartir sus experiencias. 


\section{RESUMEN}

En este trabajo se analiza la relación existente entre la práctica de actividades de ocio y las condiciones personales y de vida de la población mayor, y su influencia en la Calidad de Vida (CdV) global. Los datos proceden de una muestra representativa de 499 encuestados con 65 o más años de edad que residen en viviendas familiares de la Comunidad de Madrid. Se utilizan indicadores objetivos y subjetivos relativos a la dimensión del ocio y otros atributos de la CdV a la edad anciana, y se aplican técnicas estadísticas univariantes y bivariantes así como análisis clúster. Los resultados muestran diferentes prácticas de ocio e influencia en la $\mathrm{CdV}$ de los mayores, que varían según las características personales, el estado de salud o la forma de vida de las personas.

Palabras clave: Actividades de ocio, Calidad de Vida, población mayor, Comunidad de Madrid.

\section{ABSTRACT}

This paper aims to examinate the relationship between the practice of leisure activities and personal and living conditions of the population and its influence on global Quality of Life (QoL). Data come from a representative sample of 499 respondents with 65 years old and more in community-dwelling in the Madrid Region. Objective and subjective indicators related to leisure activities and other attributes of QoL and personal characteristics of the elderly are used. Univariate and bivariate statistical techniques are applied as well as cluster analysis. The results show different leisure practices and influence on the QoL of the elderly, in relation to personal characteristics, health status and the ways of life of the population.

Key words: Leisure activities, Quality of Life, Old people, Region of Madrid.

\section{INTRODUCCIÓN}

La Calidad de Vida (CdV) se ha convertido en las últimas décadas en un tema relavante tanto para los investigadores como para los responsables de políticas sociales, en la búsqueda y mantenimiento de la satisfacción y la felicidad de las personas (Mercer, 1994). Su importancia radica en que reduciendo las pérdidas de salud asociadas a la edad se pueden aumentar las oportunidades de vivir mejor (Fagerström et al., 2007). En concreto, el análisis de las actividades realizadas por la población en los años finales de la vida también ha devenido en un asunto de interés, puesto que es a partir de la jubilación cuando los mayores disponen de más tiempo para dedicarlo a otras actividades, distintas a la laboral, que les reportan satisfacción y bienestar en el contexto de adaptación a la vejez (Nimrod, 2007).

La relación entre $\mathrm{CdV}$ y ocio ha sido puesta de manifiesto en diversos estudios empíricos que han mostrado los enormes beneficios que la práctica de actividades de ocio tiene en edades avanzadas como fuente subjetiva de bienestar en las personas (Neal et al., 1999; Heo et al., 2010). Asimismo, la importancia de practicar actividades de ocio durante la vejez se ha relacionado con una mayor probabilidad de aumentar la satisfacción global con su vida (Menec y 
Chipperfield, 1997) o mejorar sus condiciones de vida (Silverstein y Parker, 2002). También se ha observado la probabilidad de incrementar el bienestar con la vida entre las personas que mantienen niveles de actividad comparables con los de adultos más jóvenes, e incluso entre quienes practican actividades que no realizaban anteriormente (Hooyman y Kiyak, 1996).

Los beneficios obtenidos de la práctica de actividades de ocio contribuyen a mejorar determinadas parcelas de la vida, aumentando tanto la capacidad física como la psíquica e intelectual (Schooler y Mulatu, 2001). El desarrollo de actividad física en los mayores está muy relacionado con el mantenimiento de la capacidad funcional (Clark, 1996), la reducción de enfermedades (Malmberg et al., 2005) y la desaceleración del proceso de discapacidad (Janke et al., 2008). Asimismo, su efecto se ha relacionado con la mejora del estado de salud general (físico y mental) (Culos-Reed et al., 2008; Seeman et al., 1995), la reducción del riesgo de mortalidad en todas sus causas (Lan et al., 2006) y la longevidad (Rakowski y Mor, 1992). Algunos autores han determinado el beneficio del ocio en el funcionamiento intelectual, de forma que desarrollar tareas complejas tiene un efecto positivo en procesos intelectuales y en el desenvolvimiento en situaciones difíciles (Schooler y Mulatu, 2001); en la misma línea, Wang et al. (2002) destacan los beneficios en la reducción del deterioro cognitivo en la mejora del estado psicológico (King et al., 1993), en la disminución del riesgo de depresión (Herzog et al., 1998) y de estrés (Patterson, 1996).

En cuanto a las actividades no físicas, se ha observado que también conllevan importantes beneficios en la salud; así, asistir a eventos culturales favorece la longevidad (Bygren et al., 1996), y mantener una red familiar y social de calidad tiene repercusiones favorables en la salud, en el bienestar global y en la felicidad de los mayores (Onishi et al., 2005; Nilsson et al., 2006).

En España, un estudio previo realizado en la Comunidad de Madrid (Fernández-Mayoralas et al., 2007) ha demostrado que el ocio desarrollado en el tiempo libre es la quinta dimensión de CdV más nominada por la población mayor en su definición y evaluación de CdV (38\% de las personas lo nombraron espontáneamente), por detrás de la salud (96\%), la red familiar (82\%), la situación económica (76\%) y la red social (57\%), lo que avala la importancia que la población mayor de la Comunidad de Madrid da a las actividades de ocio en su tiempo libre.

En este contexto geográfico y sociodemográfico, y a partir de los antecedentes teóricos reseñados, este artículo se centra en el análisis de la práctica de ocio entre la población mayor no institucionalizada que reside en la Comunidad de Madrid, con el propósito de identificar qué factores personales y de condiciones de vida subyacen en la participación en actividades de ocio como dimensión importante en la CdV. En primer lugar, se estudian las actividades practicadas por ese grupo de población según tipología, y se determina el nivel de satisfacción resultante de dicha práctica. Para explicar la realización de actividades de ocio se tienen en cuenta las características sociodemográficas de esta población así como otras condiciones personales relacionadas con las dimensiones más relevantes de la $\mathrm{CdV}$ como son la salud, el bienestar emocional y afrontamiento de vida, los recursos económicos y otros factores del entorno familiar y social (Fernández-Mayoralas et al., 2007; Rodríguez et al., 2011; RojoPérez et al., 2007; Prieto-Flores et al., 2008).

Se considera la existencia de diferencias en la práctica de actividades de ocio y en su contribución a la satisfacción con esta dimensión de la $\mathrm{CdV}$ de los mayores en función, sobre todo, de sus características sociodemográficas, pero también de otras condiciones como la salud y el funcionamiento, la forma de convivencia y las redes familiar y social. Así, pues, la hipótesis 
planteada parte de la idea de que en el tipo y frecuencia de ocio practicado influyen diversos factores de carácter individual, familiar y social, y que el ocio desarrollado según las circunstancias personales y contextuales contribuye a la mejora de la $\mathrm{CdV}$ dominio-específica.

\section{CONCEPTUALIZACIÓN, METODOLOGÍA Y MATERIAL UTILIZADO}

Para abordar los objetivos propuestos es necesario clarificar dos aspectos de tipo metodológico (Lloyd y Auld, 2002). El primero es la naturaleza y contenidos del ocio, por lo que es preciso previamente definir qué es, qué actividades se consideran ocio y cómo se clasifican. El ocio en la literatura científica se relaciona normalmente con el tiempo que resta después de las actividades obligatorias (educación, trabajo, tareas domésticas y cuidado personal) (Gerstl, 1983). En cuanto a la clasificación de las actividades de ocio, normalmente se distingue entre ocio activo (práctica de deportes, lectura, trabajo no remunerado, realización de tareas domésticas) y pasivo (ver la TV, vídeos, DVDs,...). Sin embargo, también la literatura científica recoge otras clasificaciones basadas en: i) actividades realizadas fuera o dentro del hogar (Neal et al., 1999), ii) en la práctica individual o colectiva o en el nivel de complejidad de las mismas (Duque y Mateo, 2008), iii) actividades centradas en las personas o en lugares (Lloyd y Auld, 2002), o iv) tipología de actividades (de socialización, creativas o artísticas, intelectuales o deportivas) (Scott y Willits, 1998). Con respecto a las personas mayores, se han observado otras agrupaciones de tipo cualitativo teniendo en cuenta nueve tipos de actividades: juegos, educación y entretenimiento cultural, deportes, naturaleza, arte, coleccionismo, artesanía, voluntariado y participación en organizaciones (Overs et al., 1977).

El segundo aspecto metodológico a considerar se basa en los criterios para medir las actividades de ocio, observándose diversas alternativas. Una aproximación es usar criterios de tipo objetivo basados en la frecuencia de realización de actividades diversas (Allen, 1991), la cantidad de tiempo libre dedicado a actividades, el acceso a equipamientos de ocio, o la frecuencia de uso de este equipamiento (Moller, 1992). Según ello, se asume que una mayor disponibilidad de tiempo libre en la vejez o tras la jubilación, una alta frecuencia en la práctica de actividades y el aumento del uso de equipamientos de ocio influyen directamente en la mejora de la $\mathrm{CdV}$ de las personas, por lo que algunos resultados del uso de este tipo de indicadores muestran una relación positiva entre el ocio y la CdV (Allen, 1991).

Sin embargo, se ha ignorado habitualmente otro criterio, el subjetivo, para tener en cuenta la valoración que la experiencia del ocio produce en las personas. Los dos enfoques (objetivo y subjetivo) tomados individualmente se consideran demasiado simplistas en la valoración de un fenómeno tan complejo. Por ello, actualmente se tienden a combinar ambas perspectivas, a pesar de los problemas de consistencia y compatibilidad detectados entre ambas (Lloyd y Auld, 2002). Esta doble aproximación ha sido seguida en este artículo con objeto de comprender el ocio como una experiencia individual en tanto que actitud hacia el ocio y satisfacción producida.

Para cubrir los objetivos propuestos en este trabajo se utilizó una encuesta sobre CdV global realizada a 499 personas como muestra representativa de la población con 65 años o más años que reside en vivienda familiar en la Comunidad de Madrid (CadeViMa-2005). La elección de Madrid responde tanto al número total de población mayor, como a la variedad de características socioeconómicas y territoriales de su población, por ser considerados en la literatura contemporánea factores que introducen diferencias en el bienestar 
y CdV de la población (Lu y Gilmour, 2004). Sobre un universo de 959.993 personas (Instituto de Estadística de la Comunidad de Madrid, 2005) se llevó a cabo un muestreo aleatorio estratificado con afijación proporcional al tamaño de cada estrato; los estratos se establecieron por sexo (hombre, mujer), edad (65-74 años, 75-84, y 85 y más) y tamaño del área de residencia, adaptado de la zonificación estadística del Instituto de Estadística de la Comunidad de Madrid (Instituto de Estadística de la Comunidad de Madrid, 2001) en siete áreas (Centro, Norte y Sur del municipio de Madrid; área metropolitana NW, E y $\mathrm{S}$; otras áreas no metropolitanas). El error permitido fue de $\pm 4 \%$, para una $p \leq 0.05$ y un nivel de confianza del $95 \%$.

La encuesta se diseñó como un cuestionario semi-estructurado con preguntas de carácter objetivo y subjetivo de las siguientes dimensiones de la CdV: salud, hogar y forma de vida, red familiar, red social, redes de apoyo, actividades de ocio, entorno residencial, bienestar emocional, creencias y valores, recursos económicos y características sociodemográficas.

Respecto a la dimensión del ocio en la $\mathrm{CdV}$ de los mayores, el cuestionario recoge información sobre la frecuencia de realización (habitualmente, algunas veces, nunca) de catorce actividades de ocio según los siguientes cinco tipos establecidos a partir de la bibliografía al uso (García-Martín et al., 2004; Silverstein y Parker, 2002), a saber:

i) ocio físico (1: actividades físicas: deportes, nadar, gimnasio);

ii) ocio cultural (2: actividades creativas; 3 : ir a centros de mayores, clubes sociales o culturales; 4: ir al cine/teatro; 5: leer (periódicos, libros...); 6: uso de nuevas tecnologías: internet; 7: cursos de manualidades);

iii) ocio participativo y social (8: salir fuera con amigos a pasear; 9: participación en ONGs, asociaciones sociales/civiles; 10: actividades organizadas por la parroquia; 11 : ir a eventos deportivos (espectador);

iv) ocio pasivo (12: oír música, ver vídeos/DVDs; 13: ver TV, oír la radio); y

v) viajes y turismo (14: viajar, turismo).

Asimismo, en el cuestionario se demanda información de las actividades que los entrevistados iniciaron en los últimos años, por cuáles muestran más interés, y las razones por las que se hacen más o menos actividades de las que le gustaría a la persona. En cuanto a la perspectiva subjetiva, se pregunta por el nivel de satisfacción con las actividades realizadas.

Para poner en relación el nivel de participación de los mayores en actividades de ocio y variables personales y contextuales, se ha hecho uso de otra información como el sexo, edad, estado civil, nivel de estudios, clase social, ingresos mensuales y satisfacción con la situación económica general (Rodríguez-Rodríguez et al., 2011), nivel de competencia relacionado con la salud y el funcionamiento (Fernández-Mayoralas et al., 2007), bienestar emocional y afrontamiento de vida (Prieto-Flores et al., 2008), y redes familiar y social (Ahmed-Mohamed y Rojo-Pérez, 2011).

Con respecto a las técnicas estadísticas aplicadas para afrontar los objetivos propuestos, inicialmente se ha realizado un análisis estadístico descriptivo sobre la frecuencia de realización de actividades de ocio. Posteriormente, con el propósito de reducir la dimensionalidad del tema tratado y facilitar su análisis, se ha hecho uso de la técnica de análisis clúster para obtener una clasificación de sujetos en grupos homogéneos según el tipo de ocio y la intensidad de su práctica, considerando la frecuencia de realización «habitual» como la que mejor discrimina en el establecimiento del perfil de sujetos según la práctica de actividades (Tabla 1). El procedimiento 
de clasificación de sujetos ha resultado de gran utilidad en diversos estudios (García-Martín et al., 2004; Silverstein y Parker, 2002; Paillard-Borg et al., 2009); en nuestro caso, se han obtenido 4 grupos homogéneos y auto-excluyentes de personas según las actividades practicadas y la frecuencia de realización de las mismas (Fig. 1). El método de clasificación utilizado ha sido el de clúster no jerárquico, recomendado para bases de datos con un elevado número de casos (Norusis, 1990). Los resultados de esta clasificación han sido validados mediante análisis discriminante al clasificar correctamente el $99,6 \%$ de los casos agrupados originales.

Figura 1

ANÁLISIS CLÚSTER PARA LA CLASIFICACIÓN DE INDIVIDUOS EN GRUPOS HOMOGÉNEOS SEGÚN LAS ACTIVIDADES DE OCIO PRACTICADAS

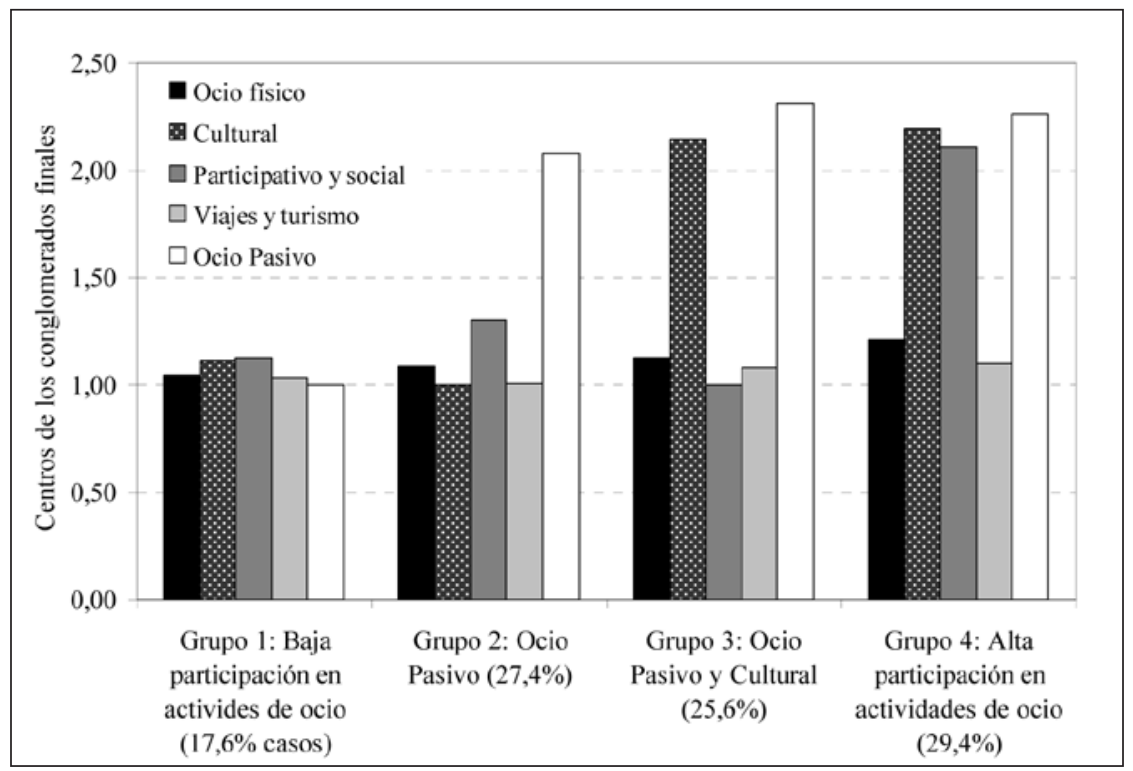

Fuente: Encuesta CadeViMa, 2005. Elaboración propia.

Así, de entre los cuatro grupos homogéneos resultantes, el primero, con un 17,6\% de casos, se caracteriza por un bajo nivel de participación en actividades de ocio de cualquier tipo; un segundo grupo $(27,4 \%)$ realiza un ocio fundamentalmente pasivo; en tercer lugar, el $25,6 \%$ de los casos se caracteriza por desarrollar actividades de ocio, sobre todo ocio pasivo, combinado con cultural; finalmente, el grupo más numeroso $(29,3 \%)$ practica indistintamente e intensamente cualquier tipo de ocio, con excepción de las actividades físicas, viajes y turismo, y con especial relevancia ocio pasivo, cultural, y participativo y social. También en los grupos 3 y 4 el ocio pasivo juega un rol reseñable. Estos resultados de tipificación de mayores según el ocio practicado han sido utilizados, posteriormente, para analizar la asociación de sujetos con variables personales y contextuales, con el objeto de conocer los factores subyacentes en la práctica de ocio como dimensión de $\mathrm{CdV}$ en la vejez.

Los grupos de sujetos resultantes de esta clasificación han sido utilizados en análisis posteriores para estudiar la posible relación con las condiciones personales y otros dominios de la 
$\mathrm{CdV}$, así como los factores subyacentes en la práctica de actividades de ocio en la vejez; para ello se han realizado tablas de contingencia, análisis de correspondencias y análisis de correlación.

\section{LAS ACTIVADES DE OCIO: CUÁLES Y CUÁNTAS PRACTICAN LA POBLACIÓN MAYOR}

De entre las actividades de ocio, la practicada con mayor frecuencia por los mayores es ver la TV y oír la radio $(95,2 \%$ de la población mayor dijo practicar estas actividades habitualmente o algunas veces), seguido por leer (periódicos, libros, ...), salir con los amigos a pasear, y escuchar música y ver vídeos/DVDs (Tabla 1). También un elevado porcentaje declaró viajar y hacer turismo $(64,6 \%)$ (habitualmente o algunas veces), aunque menos de la mitad son los que acuden al cine o al teatro, y menos todavía los que declaran realizar alguna actividad física.

Tabla 1

FRECUENCIA DE PRÁCTICA DE ACTIVIDADES DE OCIO ENTRE LA POBLACIÓN MAYOR (\%)

\begin{tabular}{|c|c|c|c|}
\hline \multirow{2}{*}{ Actividades realizadas según tipo de ocio } & \multicolumn{3}{|c|}{$\begin{array}{l}\text { Frecuencia de realización } \\
\text { (en \% sobre cada actividad) }\end{array}$} \\
\hline & Habitualmente & Algunas veces & Nunca \\
\hline \multicolumn{4}{|l|}{ Ocio físico } \\
\hline 1. Actividades físicas & 12,9 & 25,1 & 62,0 \\
\hline \multicolumn{4}{|l|}{ Ocio cultural } \\
\hline 2. Actividades creativas & 5,1 & 24,2 & 70,6 \\
\hline 3. Acudir al Club Social y otras culturales & 4,1 & 19,6 & 76,3 \\
\hline 4. Acudir al cine, teatro... & 4,3 & 44,0 & 51,6 \\
\hline 5. Leer (periódicos, libros) & 53,9 & 34,1 & 12,0 \\
\hline 6. Utilizar nuevas tecnologías: Internet & 0,6 & 7,0 & 92,3 \\
\hline 7. Cursos de manualidades & 2,9 & 9,6 & 87,5 \\
\hline \multicolumn{4}{|l|}{ Ocio y tiempo libre } \\
\hline 8. Salir con amigos a pasear & 38,0 & 44,1 & 17,9 \\
\hline 9. Participar en ONGs / Asociaciones & 1,4 & 4,1 & 94,4 \\
\hline $\begin{array}{r}\text { 10. Participar actividades organizadas por la } \\
\text { Parroquia }\end{array}$ & 3,1 & 23,4 & 73,5 \\
\hline 11. Acudir a eventos deportivos & 1,0 & 16,3 & 82,6 \\
\hline \multicolumn{4}{|l|}{ Ocio pasivo } \\
\hline 12. Escuchar música, ver vídeos/DVD & 20,8 & 45,9 & 33,3 \\
\hline 13. Ver TV, oír la radio & 80,4 & 14,7 & 4,8 \\
\hline \multicolumn{4}{|l|}{ Viajes y turismo } \\
\hline 14. Viajar, hacer turismo & 6,0 & 58,6 & 35,4 \\
\hline
\end{tabular}

Fuente: Encuesta CadeViMa, 2005. Elaboración propia. 
En cuanto a las actividades de tipo intelectual, que requieren desarrollar alguna habilidad especial, la participación es todavía menor, de forma que sólo una de cada diez personas realizó («habitualmente» o «algunas veces») algún tipo de curso (aunque sea de manualidades y trabajos prácticos), un 7,6\% utiliza nuevas tecnologías (Internet), y únicamente el 5,5\% informó pertenecer a alguna asociación u ONG. En suma, el análisis descriptivo ha mostrado que son las actividades de ocio de tipo pasivo (oír la radio, ver la TV, escuchar música) las más frecuentemente realizadas por los mayores residentes en vivienda familiar en la Comunidad de Madrid.

Tabla 2

INTENSIDAD DE LAPRÁCTICA DEL OCIO EN RELACIÓN CON LAS CARACTERÍSTICAS SOCIODEMOGRÁFICAS

\begin{tabular}{|c|c|c|c|c|c|c|c|}
\hline \multirow{3}{*}{ Variables } & \multicolumn{5}{|c|}{ Número de actividades practicadas } & \multirow{3}{*}{ Total } & \multirow{3}{*}{ Media } \\
\hline & \multirow{2}{*}{$\begin{array}{l}\text { Ninguna } \\
\text { actividad }\end{array}$} & \multirow{2}{*}{1 a 3} & \multirow{2}{*}{4 a 6} & \multirow{2}{*}{7 a 9} & \multirow{2}{*}{$\begin{array}{l}100 \\
\text { más }\end{array}$} & & \\
\hline & & & & & & & \\
\hline $\mathrm{N}$ (nº de casos válido) & 14 & 79 & 203 & 154 & 47 & 497 & Media: 5,95 \\
\hline$\%$ & 2,8 & 15,9 & 40,8 & 31,0 & 9,5 & 100,0 & Desv. Típ.: 2,66 \\
\hline Sexo & \multicolumn{5}{|c|}{ NS } & & \\
\hline Hombre & 2,5 & 12,3 & 38,9 & 35,5 & 10,8 & 204 & 6,30 \\
\hline Mujer & 3,1 & 18,4 & 42,2 & 27,9 & 8,5 & 295 & 5,71 \\
\hline Edad & \multicolumn{5}{|c|}{$(* * *)$} & & \\
\hline 65-74 años & 2,0 & 8,5 & 41,5 & 35,4 & 12,6 & 247 & 6,55 \\
\hline $75-84$ & 3,4 & 20,8 & 38,8 & 30,3 & 6,7 & 179 & 5,58 \\
\hline 85 o más & 4,1 & 28,8 & 43,8 & 17,8 & 5,5 & 73 & 4,84 \\
\hline Estado civil & \multicolumn{5}{|c|}{$(* *)(a)$} & & \\
\hline Soltero/a & & 18,8 & 68,8 & 6,3 & 6,3 & 16 & 5,06 \\
\hline Casado/a, viviendo en pareja & 2,6 & 10,6 & 44,2 & 33,6 & 9,1 & 265 & 6,15 \\
\hline Separado/a, divorciado/a & & 5,9 & 23,5 & 58,8 & 11,8 & 17 & 7,47 \\
\hline Viudo/a & 3,5 & 23,6 & 35,7 & 27,1 & 10,1 & 199 & 5,63 \\
\hline Nivel de estudios & \multicolumn{5}{|c|}{$(* * *)(b)$} & & \\
\hline No sabe leer, no sabe escribir & & 15,4 & 53,8 & 30,8 & & 13 & 5,46 \\
\hline Sin estudios, o inferiores a primarios & 0,8 & 23,8 & 46,8 & 23,0 & 5,6 & 126 & 5,41 \\
\hline Educación primaria & 5,0 & 20,9 & 39,3 & 27,9 & 7,0 & 201 & 5,44 \\
\hline $\begin{array}{r}\text { Educación secundaria ler. grado (estudios } \\
\text { terminados a los } 12-16 \text { años) }\end{array}$ & 3,2 & 5,3 & 44,2 & 32,6 & 14,7 & 95 & 6,57 \\
\hline $\begin{array}{l}\text { Educación secundaria } 2^{\circ} \text { grado, o Formación } \\
\text { Profesional (estudios terminados a los 16-18 años) }\end{array}$ & 0,0 & 0,0 & 30,6 & $\mathbf{5 0 , 0}$ & 19,4 & 36 & 7,67 \\
\hline Educación universitaria & 0,0 & 0,0 & 16,7 & 62,5 & 20,8 & 24 & 8,25 \\
\hline Clase social & \multicolumn{5}{|c|}{$(* * *)$} & & \\
\hline Baja & 0,7 & 21,6 & 48,2 & 24,5 & 5,0 & 139 & 5,48 \\
\hline Media baja & 3,4 & 17,0 & 42,1 & 29,8 & 7,7 & 235 & 5,73 \\
\hline Media & 6,0 & 9,6 & 33,7 & 32,5 & 18,1 & 83 & 6,51 \\
\hline Media alta / alta & 0,0 & 2,5 & 22,5 & 57,5 & 17,5 & 40 & 7,73 \\
\hline Satisfacción con el ocio & \multicolumn{5}{|c|}{$(* * *)$} & & \\
\hline Poco / nada / regular satisfecho/a & 1,3 & 28,8 & 45,0 & 21,3 & 3,8 & 80 & 5,06 \\
\hline Bastante & 1,7 & 12,2 & 44,1 & 33,2 & 8,8 & 295 & 6,19 \\
\hline Muy & 7,3 & 13,8 & 28,4 & 34,9 & 15,6 & 109 & 6,15 \\
\hline
\end{tabular}

Test $\chi 2$; entre paréntesis $p$-values: NS: no significativo; $*:<0,05 ; * *:<0,01 ; * *:<0,001$. En negrita, valor igual o superior a la situación media. (a) El 35,0\% de las celdas tienen una frecuencia esperada inferior a 5; la frecuencia mínima esperada es de 4,5 (b). El 36,7\% de las celdas tienen una frecuencia esperada inferior a 5; la frecuencia máxima esperada es de 3,7.

Fuente: Encuesta CadeViMa, 2005. Elaboración propia. 
De entre todas las actividades de ocio consideradas en este estudio, casi 6 de cada 10 mayores declara una práctica habitual de entre 1 y 6 actividades (media: 5,8) (Tabla 2), observándose una asociación estadísticamente significativa entre el número de actividades practicadas y la edad, el estado civil y la clase social, de forma que una mayor intensidad en la práctica de ocio se da entre los viejos-jóvenes, las personas casadas y separadas/ divorciadas, quienes han alcanzado un nivel de estudios más elevado y se agrupan en los tramos más elevados del estatus social. Respecto a la evaluación de la satisfacción con las actividades de ocio, como indicador subjetivo, más de 8 de cada 10 mayores declara estar bastante o muy satisfecho, existiendo una relación directa entre el número de actividades practicadas y la satisfacción. Por su parte, no se observa asociación estadística entre el número de actividades practicadas y el género, pero la tendencia señala una práctica más elevada entre la población masculina.

\section{LA PARTICIPACIÓN EN ACTIVIDADES DE OCIO SEGÚN FACTORES SOCIODEMOGRÁFICOS}

Algunas teorías sostienen la importancia del género en el estilo de vida y hábitos de ocio y consumo de la población (Tomlinson, 2003), tendencia que también se observa en el caso de los mayores de la Comunidad de Madrid al considerar su clasificación en grupos homogéneos o clúster. De esta forma, los hombres son más propensos a la práctica de actividades de cualquier tipo de ocio en su tiempo libre, mientras que las mujeres muestran especial dedicación a actividades de carácter pasivo o, incluso, una baja participación (Tabla 3).

La influencia de la edad en la práctica y tipo de ocio se observa con nitidez (Tabla 3), ya que a media que se envejece no sólo disminuye el número de actividades realizadas por los mayores (Tabla 2), sino que también la tipología de ocio varía claramente (Herzog et al., 1989). Las personas de 85 y más años son las que desarrollan menos actividades de ocio, ya que, como se ha visto anteriormente, realizan exclusivamente actividades de ocio pasivo (clúster 2); sin embargo, ese porcentaje se reduce a la mitad entre quienes tienen entre $65 \mathrm{y}$ 74 años, siendo así que éstos también están involucrados en actividades pasivas y culturales (clúster 3), y entre los más jóvenes es significativa su inclusión en el grupo de alta participación en actividades de ocio (clúster 4).

El estado civil de los entrevistados no revela diferencias estadísticamente significativas en cuanto a las actividades desarrolladas (Tabla 3), si bien se aprecia la tendencia a una práctica más elevada de actividades de cualquier tipo entre los casados y los separados/divorciados, a lo que se suman los solteros en actividades de tipo cultural; por otro lado, los viudos desarrollan un ocio más pasivo, quizás por la edad media más elevada de este colectivo, junto a otras posibles influencias del nivel de competencia relacionado con la salud y el funcionamiento según se envejece.

Respecto al nivel educativo, se observa una asociación estadística significativa entre la práctica de ocio distinto al pasivo y el nivel de formación (Tabla 3). De esta manera, el grupo más numeroso de los que no tienen estudios, o tienen de inferiores a primarios, o únicamente educación primaria, se incluyen en el clúster 2 (ocio pasivo) y, al contrario, los que cuentan con mayor formación (especialmente secundaria y universitaria) suelen estár más involucrados en ocio de tipo cultural. 
Tabla 3

TIPOLOGÍA DE SUJETOS MAYORES SEGÚN LA PRÁCTICA DEL OCIO Y RASGOS SOCIODEMOGRÁFICOS

\begin{tabular}{|c|c|c|c|c|c|}
\hline \multirow{3}{*}{ Variables } & \multicolumn{4}{|c|}{ Tipología de sujetos según la participación en actividades de ocio } & \multirow{3}{*}{ Total } \\
\hline & \multirow{2}{*}{$\begin{array}{l}\text { Grupo 1: Baja } \\
\text { participación en } \\
\text { actividades de ocio }\end{array}$} & \multirow{2}{*}{$\begin{array}{l}\text { Grupo 2: Ocio } \\
\text { pasivo }\end{array}$} & \multirow{2}{*}{$\begin{array}{l}\text { Grupo 3: Ocio } \\
\text { pasivo y cultural }\end{array}$} & \multirow{2}{*}{$\begin{array}{c}\text { Grupo 4: Alta } \\
\text { participación en } \\
\text { actividades de ocio }\end{array}$} & \\
\hline & & & & & \\
\hline $\mathrm{N}^{o}$ de casos válido & 88 & 137 & 128 & 146 & 499 \\
\hline$\%$ & 17,6 & 27,5 & 25,7 & 29,3 & 100,0 \\
\hline Sexo & \multicolumn{4}{|c|}{$(*)$} & \\
\hline Hombre & 17,2 & 20,6 & 27,0 & 35,3 & 204 \\
\hline Mujer & 18,0 & 32,2 & 24,7 & 25,1 & 295 \\
\hline Edad & \multicolumn{4}{|c|}{$(* *)$} & \\
\hline 65-74 años & 17,4 & 20,2 & 27,1 & 35,2 & 247 \\
\hline $75-84$ & 17,3 & 31,3 & 25,7 & 25,7 & 179 \\
\hline 85 o más & 19,2 & 42,5 & 20,5 & 17,8 & 73 \\
\hline Estado civil & \multicolumn{4}{|c|}{ NS } & \\
\hline Soltero/a & 18,8 & 31,3 & 37,5 & 12,5 & 16 \\
\hline Casado/a, viviendo en pareja & 17,7 & 24,5 & 27,5 & 30,2 & 265 \\
\hline Separado/a, divorciado/a & 17,6 & 11,8 & 17,6 & 52,9 & 17 \\
\hline Viudo/a & 17,4 & 32,3 & 22,9 & 27,4 & 201 \\
\hline Nivel de estudios & \multicolumn{4}{|c|}{$(* * *)$} & \\
\hline No sabe leer, no sabe escribir & 30,8 & 23,1 & 30,8 & 15,4 & 13 \\
\hline Sin estudios, o inferiores a primarios & 20,5 & 37,8 & 19,7 & 22,0 & 127 \\
\hline Educación primaria & 21,4 & 30,3 & 23,4 & 24,9 & 201 \\
\hline $\begin{array}{r}\text { Educación secundaria ler. grado } \\
\text { (estudios terminados a los 12-16 años) }\end{array}$ & 13,68 & 13,7 & 32,6 & 40,0 & 95 \\
\hline $\begin{array}{r}\text { Educación secundaria } 2^{\circ} \text { grado, } 0 \\
\text { Formación Profesional (estudios } \\
\text { terminados a los } 16-18 \text { años) }\end{array}$ & 0,0 & 13,9 & 36,1 & 50,0 & 36 \\
\hline Educación Universitaria & 8,0 & 24,0 & 32,0 & 36,0 & 25 \\
\hline Clase social & \multicolumn{4}{|c|}{$(* *)$} & \\
\hline Baja & 20,9 & 36,7 & 20,1 & 22,3 & 139 \\
\hline Media Baja & 18,2 & 28,0 & 24,2 & 29,7 & 236 \\
\hline Media & 14,5 & 12,0 & 36,1 & 37,3 & 83 \\
\hline Media Alta/Alta & 9,8 & 24,4 & 31,7 & 34,1 & 41 \\
\hline
\end{tabular}

Test $\chi 2$; entre paréntesis p-values: NS: no significativo; $*:<0,05 ; * *:<0,01 ; * * *:<0,001$. En negrita, valor igual o superior a la situación media.

Fuente: Encuesta CadeViMa, 2005. Elaboración propia. 
Por lo que refiere a la clase social, entendida como variable derivada del nivel de estudios y de la condición socioeconómica (Rojo-Pérez et al., 2007), sólo el 4,4\% pertenece a un estatus medio-alto, y otro 3,8\% al alto; sin embargo, el grupo de mayores clasificados como de clase baja o media-baja es el más representativo, en consonancia con otros estudios sobre población mayor (Lassey y Lassey, 2001). La mayor parte de las personas mayores integrantes del clúster 4 (alta participación en cualquier tipo de ocio) y del clúster 3 (ocio pasivo y cultural) pertenece a clases sociales media y superior, mientras que los niveles de menor actividad (clúster 1) e incluso de ocio pasivo (clúster 2) se dan entre las personas mayores de clase social inferior (Tabla 3). No obstante, y puesto que el 75\% de los entrevistados se incluye en la categoría de estatus social bajo y medio-bajo, las diferencias se minimizan, predominando quienes practicaron poco ocio o de tipo pasivo.

En relación con esta situación formativa y de estatus social, el bajo nivel de estudios de los mayores madrileños, relacionado con la pertenencia generalizada a un estatus social bajo o medio-bajo, permite comprender la escasa demanda de cursos de formación en la actualidad y el también insuficiente interés que esta población tiene por otras actividades educativas (idiomas, informática, educación artística, distintos niveles de instrucción, etc.), ya que más de 8 de cada 10 personas no declara interés, o poco, por la realización de ningún tipo de cursos.

\section{LA INFLUENCIA DE LOS RECURSOS ECONÓMICOS Y LAS CONDICIONES DE SALUD EN LA PRÁCTICA DE ACTIVIDADES DE OCIO}

Si bien la influencia que ejercen el nivel educativo y la clase social en relación con la práctica de ocio es bastante clara, no ocurre lo mismo con el nivel de ingresos de los mayores, al menos medido de forma objetiva (ingresos totales mensuales). Por un lado, la tasa de no respuesta relacionada con las preguntas sobre ingresos económicos es relativamente elevada (un 33,3\%), aunque está en la línea de la que se da a este tipo de cuestiones en otros estudios sobre población mayor (Instituto de Mayores y Servicios Sociales, 2004; Rodríguez-Rodríguez et al., 2011). Por otro lado, a pesar de que existe asociación estadística significativa (chi cuadrado: 40,695; gl: 9; p-values: 0,000), esta asociación debe ser tomada con cautela, precisamente por la tasa de no respuesta, para apoyar si una mayor disponibilidad económica se relaciona con la práctica de más ocio y con tipos de ocio determinados. Sin embargo, se observa la tendencia a una práctica de actividades de ocio más baja entre quienes declararon una menor percepción de ingresos mensuales, inferior a $600 €$, pese a que la relación no está tan clara a medida que aumentan los ingresos, tal como ya se ha señalado en otro trabajo (Rodríguez-Rodríguez et al., 2011). La gran mayoría de entrevistados dispone de una renta de entre 300 y $900 €$ mensuales, y sólo en función de sus ingresos no se perfila ninguna pauta especial en su comportamiento respecto al ocio.

A diferencia de los ingresos absolutos, resulta más relevante la satisfacción que la situación económica general de los propios mayores produce en relación con las actividades de ocio practicadas ( $p$-values 0,000 ). Por un lado, no necesariamente los mayores más satisfechos con su situación económica son los que declaran percepciones económicas mensuales más elevadas (Rodríguez-Rodríguez et al., 2011), pero aquellos bastante o muy satisfechos tienen una participación más activa en todo tipo de ocio (clúster 4) o en actividades de ocio 
pasivo y cultural (clúster 3) que quienes están menos satisfechos (Fig. 2). Por otro lado, los menos satisfechos con su situación económica tienden a desarrollar ocio más pasivo (clúster 2) o incluso a no participar en actividades de ocio.

Figura 2.

LA SATISFACCIÓN DE LOS MAYORES CON SU SITUACIÓN ECONÓMICA SEGÚN LOS GRUPOS HOMOGÉNEOS DE ACTIVIDADES DE OCIO

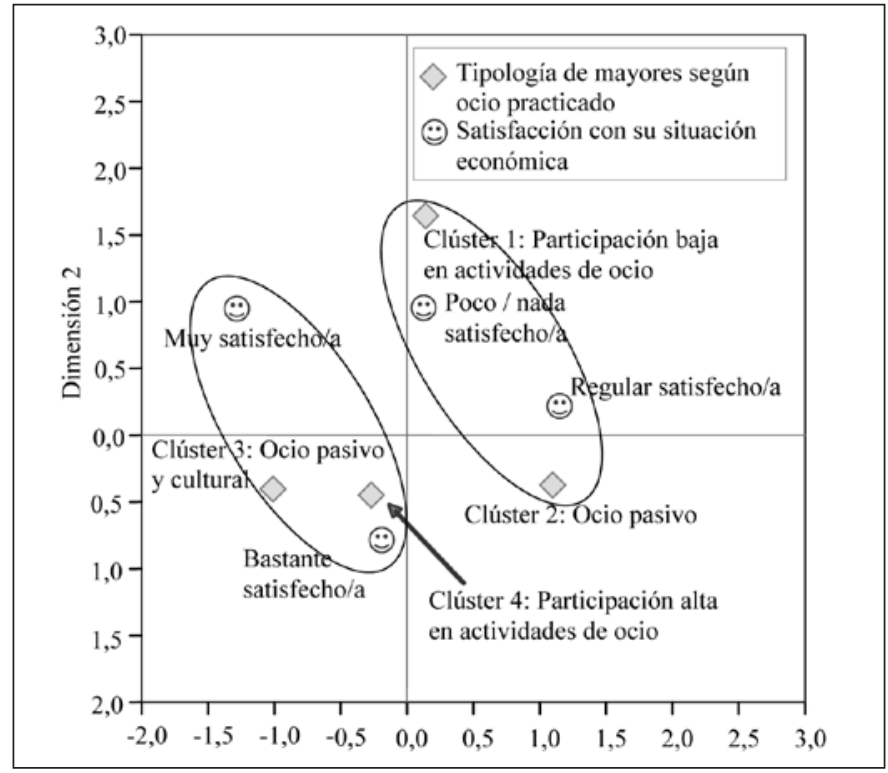

Fuente: Encuesta CadeViMa, 2005. Elaboración propia.

La salud es considerada por los propios mayores como la dimensión más importante en su CdV (Fernández-Mayoralas et al., 2007) y este hecho, unido al efecto de la práctica de ocio sobre el estado de salud general y el funcionamiento (Malmberg et al., 2005; Menec y Chipperfield, 1997; Paillard-Borg et al., 2009), justifica el análisis de la relación interdominios. En este contexto, se observa una relación significativa entre la intensidad en la práctica de actividades de ocio y la Calidad de Vida Relacionada con la Salud (CVRS) y la percepción del estado de salud, medidos ambos parámetros a partir del instrumento EuroQol-5D (The EuroQoL Group, 1990), el funcionamiento según la escala de independencia funcional (Martínez-Martín et al., 2009) y la escala de capacidad funcional de Barthel (Wade y Collin, 1988), y el nivel de depresión según el instrumento HADS-D (Zigmond y Snaith, 1983) (Tabla 4). De esta forma, quienes han declarado realizar más actividades de ocio tienen también una mejor CVRS y mejor percepción de la salud, mejor capacidad funcional y menor grado de depresión. No se ha observado correlación significativa con el número de enfermedades, si bien la tendencia parece ser inversa. Según estos resultados, un peor estado de salud general y funcionamiento estaría condicionando la práctica de actividades de ocio realizadas, seguramente por las limitaciones que dicho estado introduce. 
Tabla 4

LA INTENSIDAD EN LA PRÁCTICA DE ACTIVIDADES DE OCIO SEGÚN LA SALUD Y EL FUNCIONAMIENTO

\begin{tabular}{|l|c|}
\hline \multicolumn{1}{|c|}{ Variables de salud y funcionamiento } & $\begin{array}{c}\text { Correlación con el número de } \\
\text { actividades de ocio practicadas } \\
\text { habitualmente }\end{array}$ \\
\cline { 2 - 2 } & \begin{tabular}{c} 
r Pearson \\
\hline Calidad de Vida relacionada con la salud (EQ-5D)
\end{tabular} \\
\hline Estado de salud hoy (EQ-5D) & $0,096(*)$ \\
\hline Número de enfermedades o condiciones de salud & $-0,029(\mathrm{NS})$ \\
\hline Escala de depresión según la escala HADS-D & $-0,349(* * *)$ \\
\hline Índice de Capacidad Funcional de Barthel & $0,095(*)$ \\
\hline Escala Independencia Funcional (no incluye tareas domésticas) & $0,212(* * *)$ \\
\hline Escala Independencia Funcional (incluye tareas domésticas) & $0,125(* *)$ \\
\hline
\end{tabular}

Entre paréntesis, p-values: NS: no significativo; $*:<0,05 ; * *:<0,01 ; * * *:<0,001$.

Fuente: Encuesta CadeViMa, 2005. Elaboración propia.

Entre las principales limitaciones existe evidencia estadística significativa de que las dimensiones de condiciones de salud y funcionamiento (recogidas en el sistema descriptivo de CVRS del instrumento EuroQol-5D) (The EuroQoL Group, 1990) pueden estar influyendo en la participación activa de los mayores en su tiempo de ocio (Tabla 5), de forma que las personas que dicen tener alguna o muchas dificultades para caminar, para el cuidado personal, y para la realización de las actividades cotidianas así como estar moderadamente o muy ansioso, ven limitada su práctica de ocio y se clasifican mayoritariamente en el grupo definido por desarrollar más ocio pasivo; por el contrario, aquellas personas mayores que declaran no tener problemas en las actividades cotidianas investigadas o no mostrar ansiedad/depresión se agrupan en el clúster 4 (alta participación en actividades de ocio).

El bienestar psíquico y el sentimiento de felicidad y utilidad también han sido estudiados en relación con las actividades de ocio (Heyl et al., 2005), de forma que un estado de ánimo y una actitud positivos ante la vida son factores que predisponen a realizar determinadas actividades y especialmente las que requieren mayor esfuerzo físico; también las emociones positivas y el bienestar experimentado en el ocio son elementos que contribuyen a la CdV (Iwasaki, 2007). En este contexto, y puesto que el dominio emocional se ha revelado como causa y efecto de la salud entre la población mayor residente en vivienda familiar en la Comunidad de Madrid (Prieto-Flores et al., 2008), en este estudio se indaga sobre la posible influencia del bienestar emocional en la realización de actividades de ocio. Para ello se ha considerado un conjunto de variables latentes o componentes principales, a saber: emociones positivas (donde se agrupan las variables que miden la frecuencia con la que un individuo se siente útil, seguro 
de sí mismo, ilusionado y feliz), emociones negativas (en donde correlacionan las variables que informan sobre la frecuencia con la que un individuo se siente intranquilo o nervioso, aburrido-apático, deprimido o triste) y soledad (frecuencia de sentirse solo/a).

Tabla 5

TIPOLOGÍA DE SUJETOS MAYORES SEGÚN LA PRÁCTICA DEL OCIO Y CONDICIONES DE SALUD Y FUNCIONAMIENTO

\begin{tabular}{|c|c|c|c|c|c|}
\hline \multirow{3}{*}{ Variables } & \multicolumn{4}{|c|}{ Tipología de sujetos según la participación en actividades de ocio } & \multirow{3}{*}{ Total } \\
\hline & \multirow[t]{2}{*}{$\begin{array}{c}\text { Grupo 1: Baja } \\
\text { participación en } \\
\text { actividades de ocio }\end{array}$} & \multirow[t]{2}{*}{$\begin{array}{l}\text { Grupo 2: Ocio } \\
\text { pasivo }\end{array}$} & \multirow[t]{2}{*}{$\begin{array}{l}\text { Grupo 3: Ocio } \\
\text { pasivo y cultural }\end{array}$} & \multirow[t]{2}{*}{$\begin{array}{l}\text { Grupo 4: Alta } \\
\text { participación } \\
\text { en actividades } \\
\text { de ocio }\end{array}$} & \\
\hline & & & & & \\
\hline $\mathrm{N}$ (nº de casos válido) & 88 & 137 & 128 & 146 & 499 \\
\hline$\%$ & 17,6 & 27,5 & 25,7 & 29,3 & 100,0 \\
\hline EQ-5D-Movilidad & \multicolumn{4}{|c|}{$(* * *)$} & \\
\hline No tengo problemas para caminar & 18,4 & 22,7 & 25,9 & 32,9 & 374 \\
\hline $\begin{array}{r}\text { Tengo algunos o muchos problemas para } \\
\text { caminar }\end{array}$ & 14,6 & 42,3 & 25,2 & 17,9 & 123 \\
\hline EQ-5D-Cuidado personal & \multicolumn{4}{|c|}{$(* *)$} & \\
\hline No tengo problemas & 17,7 & 25,8 & 26,0 & 30,5 & 462 \\
\hline Tengo algunos o muchos problemas & 14,3 & 51,4 & 22,9 & 11,4 & 35 \\
\hline EQ-5D-Actividades cotidianas & \multicolumn{4}{|c|}{$(* * *)$} & \\
\hline No tengo problemas & 18,4 & 24,3 & 25,3 & 32,0 & 403 \\
\hline $\begin{array}{r}\text { Tengo algunos problemas o soy incapaz } \\
\text { de realizar mis actividades cotidianas }\end{array}$ & 14,9 & 47,3 & 25,7 & 12,2 & 74 \\
\hline EQ-5D-Dolor, malestar & \multicolumn{4}{|c|}{ (NS) } & \\
\hline No tengo dolor ni malestar & 19,8 & 23,8 & 24,5 & 31,9 & 273 \\
\hline $\begin{array}{r}\text { Tengo moderado o mucho dolor o } \\
\text { malestar }\end{array}$ & 14,7 & 32,1 & 27,2 & 25,9 & 224 \\
\hline EQ-5D-Ansiedad, depresión & \multicolumn{4}{|c|}{$(* *)$} & \\
\hline No estoy ansioso ni deprimido & 16,6 & 24,9 & 26,2 & 32,2 & 397 \\
\hline $\begin{array}{r}\text { Estoy moderadamente o muy ansioso o } \\
\text { deprimido }\end{array}$ & 21,2 & 38,4 & 23,2 & 17,2 & 99 \\
\hline
\end{tabular}

Test $\chi 2$; entre paréntesis $p$-values: NS: no significativo; $*:<0,05 ; * *:<0,01 ; * * *:<0,001$. En negrita, valor igual o superior a la situación media.

Fuente: Encuesta CadeViMa, 2005. Elaboración propia.

Estas variables latentes de la satisfacción con las capacidades personales y con el trato recibido por parte de la sociedad han sido utilizadas según la metodología aplicada en PrietoFlores et al. (2008): i) la satisfacción con las capacidades personales exteriores (que incluye 
la posición y reconocimiento por parte de la sociedad, el respeto de los demás, la apariencia física), ii) la satisfacción con las capacidades personales internas (tomar decisiones, libertad para expresar pensamientos), y iii) frecuencia con la que se siente solo/a. Sobre esta base, las emociones positivas y la satisfacción con las capacidades personales (Tabla 6) tienen una relación directa con el ocio, mientras que es inversa en cuanto al sentimiento de soledad, de manera que quienes declaran sentirse frecuentemente felices, ilusionados, con ganas de hacer cosas, o no se sienten frecuentemente solos, así como aquellos que perciben una buena imagen de sí mismos y reciben un buen trato y respeto de la sociedad, se clasifican entre los que más ocio practican. La relación inversa entre la práctica de ocio y las emociones negativas (frecuencia con que la persona mayor se siente intranquila/nerviosa; aburrida/apática o deprimida/triste) no muestra asociación estadística significativa.

Tabla 6

CORRELACIÓN ENTRE LA TIPOLOGÍA DE MAYORES SEGÚN LA PRÁCTICA DE OCIO Y BIENESTAR EMOCIONAL Y AFRONTAMIENTO DE VIDA

\begin{tabular}{|l|c|}
\hline \multicolumn{1}{|c|}{ Variables sobre bienestar emocional y afrontamiento de vida } & $\begin{array}{c}\text { Correlación con el número de } \\
\text { actividades de ocio practicadas } \\
\text { habitualmente }\end{array}$ \\
\cline { 2 - 3 } & \begin{tabular}{c} 
r Pearson \\
\hline Emociones positivas (frecuencia con la que se siente util, seguro, ilusionado, feliz)
\end{tabular} \\
\hline Emociones negativas (frecuencia con la que se siente intranquilo, aburrido, deprimido) & $0,308(* * *)$ \\
\hline Soledad (frecuencia con la que se siente solo) & $-0,012$ (NS) \\
\hline $\begin{array}{l}\text { Satisfacción con las capacidades personales exteriores (posicición-reconocimiento en la } \\
\text { sociedad, respeto de los demás, apariencia física) }\end{array}$ & $-0,157(* * *)$ \\
\hline $\begin{array}{l}\text { Satisfacción con las capacidades personales interiores (tomar decisiones, libertad para } \\
\text { expresar pensamientos) }\end{array}$ & $0,239(* * *)$ \\
\hline
\end{tabular}

Entre paréntesis, p-values: NS: no significativo; *: $<0,05 ; * *:<0,01 ; * * *:<0,001$.

Fuente: Encuesta CadeViMa, 2005. Elaboración propia.

\section{OTROS FACTORES QUE INFLUYEN EN LA PRÁCTICA DE ACTIVIDADES DE OCIO: LAS REDES FAMILIAR Y SOCIAL}

La familia es otra de las dimensiones que se ha revelado como principal de la CdV de los mayores (Rojo-Pérez et al., 2008; Fernández-Mayoralas, et al., 2007; Lassey y Lassey, 2001), y dentro de esta dimensión, la forma de convivencia o la estructura del hogar parece tener un efecto importante en la práctica de ocio, tanto en el número y tipo de actividades, como en la frecuencia con que se practican. Estudios previos demuestran que conviene distinguir entre las personas que viven solas y las que conviven con su pareja o con otros familiares (Avramov y Maskova, 2003). De entrada, no es significativo que las personas que viven solas entre los mayores de la Comunidad de Madrid hagan menos actividades de ocio que las que conviven en pareja o con familiares; sin embargo, parece que las diferencias están en el tipo de ocio: así, la característica dominante en la población analizada es que quienes viven solos o viven en pareja son los más involucrados en actividades de ocio de cualquier 
tipo (clúster 2, 3 y 4); vivir bajo otras estructuras familiares (en pareja y otros familiares, sin pareja pero con hijos, o hijos políticos, o nietos) se asocia con una baja participación en actividades de ocio (clúster 1) o con ocio pasivo y cultural (clúster 3), mientras que vivir con familiares no de primer grado es la característica más común de quienes practican ocio pasivo o pasivo y cultural (Tabla 7). Por su lado, la frecuencia de relaciones con familiares es elevada (el 64\% de los mayores tiene relaciones con familiares todos o casi todos los días), no hallándose asociación estadística entre este hecho y la práctica de ocio (Tabla 7).

Tabla 7

GRUPOS DE MAYORES (POR TIPOLOGÍA DE OCIO E INTENSIDAD DE PRÁCTICA) SEGÚN CARACTERÍSTICAS DEL HOGAR DE RESIDENCIA Y RED FAMILIAR Y SOCIAL

\begin{tabular}{|c|c|c|c|c|c|}
\hline \multirow{3}{*}{ Variables } & \multicolumn{5}{|c|}{ Tipología de sujetos según la participación en actividades de ocio } \\
\hline & \multirow{2}{*}{$\begin{array}{c}\text { Grupo 1: Baja } \\
\text { participación en } \\
\text { actividades de ocio }\end{array}$} & \multirow{2}{*}{$\begin{array}{l}\text { Grupo 2: } \\
\text { Ocio pasivo }\end{array}$} & \multirow{2}{*}{$\begin{array}{l}\text { Grupo 3: } \\
\text { Ocio pasivo y } \\
\text { cultural }\end{array}$} & \multirow{2}{*}{$\begin{array}{c}\text { Grupo 4: Alta } \\
\text { participación en } \\
\text { actividades de ocio }\end{array}$} & \multirow{2}{*}{ Total } \\
\hline & & & & & \\
\hline Número de casos & 88 & 137 & 128 & 146 & 499 \\
\hline$\%$ & 17,6 & 27,5 & 25,7 & 29,3 & 100,0 \\
\hline $\mathrm{N}^{0}$ personas hogar (min: 1; máx: 6; media: 1,9) & \multicolumn{4}{|c|}{ (NS) } & \\
\hline 1 & 15,6 & 33,8 & 20,0 & 30,6 & 160 \\
\hline 2 & 16,6 & 27,0 & 27,4 & 29,0 & 259 \\
\hline 3 o más & 25,0 & 16,3 & 31,3 & 27,5 & 80 \\
\hline Estructura del hogar & \multicolumn{4}{|c|}{$(*)$} & \\
\hline Viviendo solo/a & 15,6 & 33,8 & 20,0 & 30,6 & 160 \\
\hline Viviendo en pareja & 16,0 & 26,2 & 26,7 & 31,1 & 206 \\
\hline $\begin{array}{r}\text { En pareja, otros familiares (hijos, otros), } \\
\text { empleado, otros }\end{array}$ & 18,4 & 16,3 & 36,7 & 28,6 & 49 \\
\hline Viviendo con hijo/a, o yerno/nuera, o nieto/a & 29,9 & 19,4 & 23,9 & 26,9 & 67 \\
\hline Viviendo con otros familiares, empleado/a, otros & 5,9 & 47,1 & 41,2 & 5,9 & 17 \\
\hline Frecuencia relaciones con algún familiar & \multicolumn{4}{|c|}{ (NS) } & \\
\hline 1 ó 2 veces al mes / con menor frecuencia & 16,3 & 16,3 & 30,2 & 37,2 & 43 \\
\hline 1 ó 2 veces por semana & 19,7 & 23,5 & 24,2 & 32,6 & 132 \\
\hline Todos o casi todos los días & 16,4 & 30,2 & 26,0 & 27,3 & 311 \\
\hline Frecuencia relaciones con los amigos/as & \multicolumn{4}{|c|}{$(* *)$} & \\
\hline 1 ó 2 veces al mes / con menor frecuencia & 21,3 & 32,6 & 36,0 & 10,1 & 89 \\
\hline 1 ó 2 veces por semana & 20,0 & 25,4 & 27,7 & 26,9 & 130 \\
\hline Todos o casi todos los días & 15,4 & 26,6 & 21,2 & 36,7 & 259 \\
\hline
\end{tabular}

Test $\chi 2$; entre paréntesis p-values: NS: no significativo; *: $<0,05 ; * *:<0,01 ; * * *:<0,001$. En negrita, valor igual o superior a la situación media. Fuente: Encuesta CadeViMa, 2005. Elaboración propia. 
Respecto a la importancia de las amistades y las relaciones sociales en la $\mathrm{CdV}$, la mayoría de las personas mayores entrevistadas en la Comunidad de Madrid dicen tener más amigos que simplemente conocidos, y la frecuencia con que los ven es bastante elevada: más de la mitad $(54,2 \%)$ dice verlos prácticamente todos los días, frente al 27,2\% que los ve semanalmente, o el 18,6\% que dice verlos una o dos veces al mes (Tabla 7), lo cual rebate la idea generalizada de que los mayores viven sus vidas de forma más aislada que el resto de la población (Gauthier y Smeeding, 2003).

No hay evidencia estadísticamente significativa entre no tener amigos (este grupo sólo representa el $2,2 \%$ de la muestra) y la práctica de ocio según tipos. Por el contrario, la asociación entre la frecuencia de relaciones con amigos y el ocio es significativa, de forma que quienes declaran un escaso contacto con su red de amistad aparecen más representados en niveles bajos de actividad (clúster 1) y en actividades de tipo pasivo (clúster 2). En cambio, a medida que aumenta la frecuencia de los contactos, también lo hace la práctica generalizada de todo tipo de ocio (Tabla 7).

\section{DISCUSIÓN}

En este artículo se ha analizado la relación entre las actividades de ocio practicadas y las características y condiciones de vida de la población mayor de 65 años en el contexto de la satisfacción con el ocio practicado como dimension de la CdV. Este estudio empírico realizado en la Comunidad de Madrid ha demostrado que el ocio desarrollado en el tiempo libre es la quinta dimensión de $\mathrm{CdV}$ más nominada por la población mayor en su definición y evaluación de este constructo, y que la dimensión de ocio ocupa igualmente el quinto lugar en cuanto al nivel de satisfacción (con un valor de 60 sobre 100). En la misma línea, otros trabajos han destacado que la salud es el principal componente de la CdV de los mayores (Sotgiu et al., 2011) y que las actividades de entretenimiento están por detrás. Todo ello permite comprobar la relativa trascendencia que las actividades de ocio tienen en la calidad y condiciondes de vida de los mayores, al tener en cuenta la relación directa existente entre el número de actividades practicadas, su tipología y la satisfacción que su práctica produce. Se trata de actividades que generan a estas personas bastante o mucha satisfacción, lo cual constituye una pauta habitual en este tipo de estudios (Brajsa-Zganec et al., 2011; Lu y Hu, 2005).

Los resultados de esta investigación muestran que el ocio que los mayores dicen practicar es fundamentalmente de tipo pasivo y no requiere de grandes esfuerzos físicos ni intelectuales. Además, el ocio se convierte en más pasivo a media que aumenta la edad de la persona, lo cual lleva a relacionar el posible efecto del estado de salud en la práctica y en el tipo de actividades. Esta práctica de más actividades pasivas en los mayores ha sido señalada en numerosos trabajos referidos a este colectivo demográfico (Del Barrio et al., 2009; Instituto de Mayores y Servicios Sociales, 2004) y ello implica una limitada utilización del tiempo libre y, en muchos casos, enfocada a actividades como ver la televisión, escuchar la radio, leer y, en menor medida, pasear o hacer ejercicio físico moderado (CIS, 2007).

El carácter pasivo sugiere que sólo una pequeña proporción de mayores están «utilizando» de forma activa sus años de retiro, en cuanto a niveles de participación, número y tipo de actividades realizadas. Sin embargo, este resultado ha de ser contextualizado en el 
sentido de que la mayoría de estas personas continúan practicando las mismas actividades que habían realizado durante su vida, y que pocos hacen más actividades de las que venían haciendo antes de la jubilación (Nimrod, 2008). Por eso llama la atención que el 64\% de los encuestados declare hacer turismo (habitualmente o a menudo), cuando, según un informe de la participación social de los mayores (Duque y Mateo, 2008), solo el 13,7\% de las personas turistas tiene más de 65 años en España.

Otros estudios también han destacado el carácter pasivo de las actividades de ocio en este grupo etario, detectando que se pasa mucho tiempo viendo la TV durante la vejez (BrajsaZaganec et al., 2001; Jones, 1990) y que en general, el ocio es más pasivo a medida que aumenta la edad (Lefrançois et al., 1997; Paillard-Borg et al., 2009). Igualmente, Avramov y Maskova (2003) destacan que, en los países europeos, los mayores ven la TV una media de tres horas diarias, lo cual se convierte en la actividad preferida, junto con oír la radio. También la OECD (2000) señala que la mayor parte del tiempo libre tras el retiro no se usa activamente, y que todavía son muchos los obstáculos institucionales para la activación económica destinada a la población mayor. Estos datos toman sentido a partir de la teoría de la socialización (O’Brien Cousins, 1995), que mantiene que el comportamiento y las relaciones sociales varían según el género, edad y clase social.

El hecho de que la mayoría de las personas mayores de la Comunidad de Madrid no estén usando activamente su vejez, en cuanto a cómo invierten su tiempo, hay que comprenderlo en el contexto de una generación de españoles cuyas condiciones de trabajo y de vida han sido muy diferentes a las actuales, ya que muchas de estas personas mayores desarrollaron trabajos manuales durante su vida profesional, tuvieron un bajo nivel educativo, y escasas aficiones de tipo cultural e intelectual.

En el caso de los mayores madrileños, los factores influyentes en el tipo y frecuencia de actividades de ocio y tiempo libre practicadas son de tipo individual, familiar y social, tal y como se apuntaba en las asunciones planteadas, y como también han destacado Avramov y Maskova (2003). Otros trabajos, como el realizado entre la población mayor y ubana en Suecia (Paillard-Borg et al., 2009) han revelado que determinadas variables como tener más edad, ser mujer, tener menor nivel educativo o relaciones sociales pobres, sufrir problemas mentales y tener limitaciones físicas, muestran correlación con realizar menos actividades de ocio.

Así, los análisis realizados muestran la tendencia de los hombres a practicar más actividades, y de tipo más activo y cultural que las mujeres, mientras que éstas se agrupan mayormente en el ocio de tipo pasivo. La diferente distribución de actividades de ocio por sexo se hace también patente no sólo para el caso español (Instituto de Mayores y Servicios Sociales, 2004), sino en otros contextos internacionales (Brajsa-Zganec et al., 2011; Paillard-Borg et al., 2009). Estas diferencias podrían ser explicadas por el hecho de que las mujeres de esas generaciones, a medida que cumplen años, se dedican más a tareas domésticas y de cuidado personal, mientras que es más habitual que los hombres practiquen actividades distintas, como caminar o ir en bicicleta, realizar otros deportes moderados, jardinería y diversas actividades relacionadas con la reparación de la vivienda (Avramov y Maskova, 2003).

Igualmente, se ha podido corroborar la influencia del nivel de estudios y de la clase social de pertenencia en el ocio, tal y como otros autores han demostrado (Lassey y Lassey, 2001), en particular al relacionar la práctica de deportes y actividades físicas con personas 
de bajo nivel educativo (Heikkinen et al., 1987), o considerando únicamente población urbana (Paillard-Borg et al., 2009). Es característico el bajo nivel educativo entre la polación mayor, ya que la mayoría, o tiene únicamente estudios primarios, o no tiene estudios, lo cual ocurre también en países de nuestro entorno cultural como en Italia, frente a otros europeos en los que se registran mayores niveles de formación entre la población mayor (Fagerström et al., 2007). Formación y clase social son, pues, características que permiten distinguir claramente la práctica de ocio más activo, corroborando, así, la hipótesis de que los individuos situados en la parte superior de la escala social tendrían más libertad que el resto para elegir dónde residir y qué hacer en su tiempo libre (lo cual se traduce en un ocio más activo y en la práctica de más actividades); por el contrario, quienes tienen un estatus más bajo también tienen más restringida la práctica de ocio o ésta se caracteriza por ser de tipo pasivo.

Sin embargo, no se ha hallado influencia directa entre la práctica de ocio y los recursos económicos, contradiciendo así la teoría general de que los ingresos facilitan o limitan, en su caso, la elección de las activades de ocio (Bittman, 1998). A lo sumo, se podría afirmar que el ocio se relaciona con los ingresos considerando la satisfacción con la percepción de la situación económica de los mayores pero sólo hasta que se dispone de un mínimo económico determinado y suficiente para vivir; sin embargo, en personas con ese mínimo cubierto, los aspectos económicos no parecen tener relación importante con las actividades practicadas (Smith, 2000).

En cuanto a las condiciones de salud, muchas veces asociadas con la edad, parecen ser los factores que más influencia ejercen tanto en la intensidad de la práctica de ocio como en su tipo (menos, y más pasivo al aumentar la edad). Además, un sentimiento positivo como sentirse optimista, feliz, útil y con ganas de hacer cosas es lo que más predispone a involucrarse en distintas actividades. Moragas (2001) señala que la salud, junto con el nivel de estudios y la clase social, son los factores más importantes para que el jubilado realice actividades ocupacionales (de carácter manual-intelectual), de forma que a menor salud, educación y clase social, mayor es la dificultad para dedicarse a cualquier tipo de actividad nueva, aunque pueden mantenerse las anteriores.

Otro hallazgo es la baja participación de los mayores en el uso y manejo de tecnologías de información y comunicación, en línea con otros estudios donde se observa que, la mitad de la población de más edad (de 65 a 74 años) utiliza el teléfono móvil de manera habitual, pero solo un $12,8 \%$ declara haber utilizado alguna vez el ordenador y un 7,9\% Internet (INE, 2007). Estos datos demuestran que España se encuentra todavía retrasada en la utilización que las personas mayores hacen de las nuevas tecnologías, y contrastan con el mayor uso en el manejo de ordenadores e Internet detectado en otros países (Kubeck et al., 1999).

El escaso interés en actividades de formación y educativas también aparece como una característica generalizada en las personas mayores en esta investigación, al igual que se ha puesto de relevancia en otros estudios internacionales (Lassey y Lassey, 2001). Únicamente los mayores de unos pocos países del mundo (norteamericanos, suecos y holandeses) aportan cifras significativas en cuanto a tiempo invertido en formación y educación (Gauthier y Smeeding, 2003), por lo que la escasa demanda de cursos a edades avanzadas estaría asociada a factores como el nivel educativo, la salud y los ingresos, pero también a otros de tipo cultural. 


\section{CONCLUSIONES}

El análisis realizado sugiere que, hoy por hoy, el retiro y el envejecimiento activo son dos conceptos que no van de la mano, aunque de cara al futuro es previsible que las actividades que realicen los mayores en su jubilación varíen a medida que se vayan incorporando nuevas cohortes de trabajadores más educados, con mayor nivel económico y con deseos de vivir la etapa de la jubilación activamente, y no sólo como un descanso después del trabajo. Por ello, y en relación a los comportamientos por edades, cada vez se reconoce más abiertamente que la vejez constituye, en la sociedad actual, una etapa activa de la vida (Hirvensalo et al., 1998). En el caso de la práctica de deportes, se considera que está cambiando la opinión de que la vejez se asocia únicamente a una etapa de descanso típica de sociedades industriales (O’Brien Cousins, 1995), para extenderse el paradigma apoyado en el deporte y en la actividad para un envejecimiento más saludable, en la línea de asociar la vejez a un período cada vez más positivo de la vida (Sotgiu et al., 2011). Además, otros factores como las mejoras constantes en la salud, la educación y la mayor esperanza de vida, permitirán aumentar la heterogeneidad de este grupo demográfico y sus oportunidades en la vejez. Quizás por eso se tenga que ir más allá del concepto de «tercera edad», y destacar el considerable potencial que el tiempo de ocio tendrá para incrementar la $\mathrm{CdV}$ de los mayores en aquellos que tengan buena salud y suficiente vigor y curiosidad intelectual.

En este contexto, uno de los retos pendientes de las futuras investigaciones es conocer a escala internacional los diferentes factores que influyen en la $\mathrm{CdV}$ de los mayores (Fagerström et al., 2007), considerando la influencia de los distintos contextos culturales (Iwasaki, 2007), de los incentivos concretos de cada país y del papel institucional, por ejemplo, en la participación de actividades y en la demanda de las de tipo formativo.

Esas diferencias culturales explican que en los países del norte de Europa la proporción de personas mayores en formación sea mucho elevada que en los países del sur del continente (Avramov y Maskova, 2003), al igual que las diferencias entre las que realizan habitualmente actividades de tipo mental o intelectual (Paillard-Borg et al., 2009). Las ratios de participación en esos países muestran que los nórdicos, más que otras nacionalidades, facilitan y favorecen un clima institucional más idóneo para promover desarrollos educativos durante la vejez.

En el mismo sentido, eso explica también que en países con mayor sensibilidad social o con mayor incidencia demográfica de las personas mayores en el conjunto de la población, su participación social sea más alta. En dichos países, los ancianos tienen menos inhibiciones y manifiestan sus deseos sin los obstáculos característicos de las sociedades latinas más conservadoras, que han asignado hasta hace poco a cada edad de la vida un papel social cerrado (Moragas, 2001); por eso las normas sociales pueden estar influyendo en la dedicación del tiempo a unas u otras actividades, además de las preferencias de la población, las oportunidades y la oferta de actividades.

Muchos de los mayores entrevistados en Madrid responden a una generación que no tuvo ni la necesidad ni las oportunidades de incrementar su formación, lo cual convierte a los modelos culturales y al estilo de vida en una explicación simple y lineal de la práctica de actividades de ocio (McPherson y Yamaguchi, 1995). De la misma forma, tampoco todos los mayores que viven en el mismo país o entorno de bienestar invierten su tiempo de la misma 
manera (Gauthier y Smeeding, 2003), lo que demuestra que en la explicación no solo son importantes los factores «macro» sino también los «micro». Como declara Nimrod (2008), la tendencia a la innovación en las actividades de ocio y la satisfacción producida entre las personas recientemente jubiladas depende de la cultura, aunque son cuestiones que requieren más investigación.

\section{BIBLIOGRAFÍA}

AHMED-MOHAMED, K. y ROJO-PÉREZ, F. (2011): «Forma de convivencia y redes familiar y de amistad» en Calidad de vida y envejecimiento: la visión de los mayores sobre sus condiciones de vida (Rojo-Pérez, F. y Fernández-Mayoralas, G., eds.). Madrid, Edit. Fundación BBVA, 169-197.

ALLEN, L. (1991): «Benefits of leisure services to community satisfaction» en Benefits of Leisure (Driver, B.L, Brown, P.J. y Peterson, G.L., eds.). State College, Pennsylvania, Edit. Venture Publishing Inc., 332-350.

AVRAMOV, D. y MASKOVA, M. (2003): Active Ageing in Europe. Population Series, vol. $1, n^{\circ}$ 41. Strasbourg. Council of Europe.

BITTMAN, M. (1998): «Social Participation and Family Welfare: The money and time costs of leisure» en Changing families, challenging futures. 6th Australian Institute of Family Studies Conference. Melbourne 25-27 November. Disponible en:

http://www.aifs.gov.au/conferences/aifs6/bittman.htm

BRAJSA-ZGANEC, A., MERKAS, M. y SVERKO, I. (2011): «Quality of Life and Leisure Activities: How do Leisure Activities Contribute to Subjective Well-Being?». Social Indicators Research, vol. 102, $\mathrm{n}^{\circ} 1,81-91$.

BYGREN, L.O., KONLAAN, B.B. y JOHANSSON, S.E. (1996): «Attendance at cultural events, reading books or periodicals, and making music or singing in a choir as determinants for survival: Swedish Interview Survey of Living Conditions». British Medical Journal, vol. 313, no 7072, 1577-1580.

CIS (2007): Barómetro, 17 marzo, n 2.681. Madrid. Centro de Investigaciones Sociológicas.

CLARK, D.O. (1996): «The effect of walking on lower body disability among older blacks and whites». American Journal of Public Health, vol. 86, $\mathrm{n}^{\circ}$ 1, 57-61.

CULOS-REED, S.N., STEPHENSON, L., DOYLE-BAKER, P.K. y DICKINSON, J.A. (2008): «Mall Walking as a Physical Activity Option: Results of a Pilot Project». Canadian Journal on Aging / La Revue Canadienne du Vieillissement, vol. 27, $\mathrm{n}^{\mathrm{o}} 1$, 81-87.

DEL BARRIO TRUCHADO, E., SANCHO CASTIELLO, Ma'T., PÉREZ ORTIZ, L. y ABELLÁN, A. (2009): «Vida cotidiana, actitudes, valores y emociones en la vejez» en Las personas mayores en España. Datos estadísticos estatales y por Comunidades Autónomas. Informe 2008, Cap. 6, Tomo I. Colección Documentos, Serie Documentos Estadísticos, $\mathrm{n}^{\circ}$ 22019. Madrid. Instituto de Mayores y Servicios Sociales (IMSERSO).

DUQUE, J.M. y MATEO, A. (coords.) (2008): La participación social de las personas mayores. Colección Estudios, Serie Personas Mayores, $n^{\circ}$ 11005. Madrid. Instituto de Mayores y Servicios Sociales (IMSERSO). 
FAGERSTRÖM, C., BORG, C., BALDUCCI, C., BURHOLT, V., WENGER, C.G., FERRING, D., WEBER, G., HOLST, G. y HALLBERG, I.R. (2007): «Life Satisfaction and Associated Factors Among People Aged 60 Years and Above in Six European Countries». Applied Research in Quality of Life, vol. 2, n 1, 33-50.

FERNÁNDEZ-MAYORALAS, G., ROJO-PÉREZ, F., PRIETO-FLORES, Mª., LEÓNFLORES, B., MARTÍNEZ-MARTÍN, P., FORJAZ, M.J., FRADES-PAYO, B. y GARCÍA, C. (2007): El significado de la salud en la calidad de vida de los mayores. Informes Portal Mayores, $n^{\circ} 74$. Madrid. Portal Mayores. Disponible en http://www.imsersomayores.csic.es/documentacion/biblioteca/registro.htm?id=52203

GARCÍA-MARTÍN, M.A., GÓMEZ-JACINTO, L. y MARTIMPORTUGUÉSGOYENECHEA, C.A. (2004): «Structural Model of the Effects of Organized Leisure Activities on the Well-Being of Older Adults in Spain». Activities, Adaptation \& Aging, vol. $28, n^{\circ} 3,19-34$.

GAUTHIER, A.H. y SMEEDING T.M. (2003): «Time use at older ages». Research on Aging, vol. 25, $\mathrm{n}^{\mathrm{o}} 3$, 247-274.

GERSTL, J. (1983): «The fine art of leisure». Management World, $\mathrm{n}^{\circ}$ 12, 8-10.

HEIKKINEN, E., KARHU, I. y JOKELA, J. (1987): «Physical exercise and related factors among elderly people in four European localities» en Physical Activity, Aging and Sports, vol. 1 (Harris, S., Heikkinen, E. y Harris, W.S., eds.). Albany, NY, Edit. Center for the Study of Aging, 157-177.

HEO, J., LEE, Y., McCORMICK, B.P. y PEDERSEN, P.M. (2010): «Daily experience of serious leisure, flow and subjective well-being of older adults». Leisure Studies, vol. 29, $n^{\circ} 2,207-225$.

HERZOG, A.R., KAHN, R.L., MORGAN, J.N., JACKSON, J.S. y ANTONUCCI, T.C. (1989): «Age differences in productive activities». Journals of Gerontology, vol. 44, $\mathrm{n}^{\circ}$ 4, 129-138.

HERZOG, A.R., MARKUS, H.R., FRANKS, M.M. y HOLMBERG, D. (1998): «Activities and well-being in older age: effects of self-concept and educational attainment». Psychology and Aging, vol. 13, $\mathrm{n}^{\circ} 2,179-185$.

HEYL, V., WAHL, H.-W. y MOLLENKOPF, H. (2005): «Vision, out-of-home activities, and emotional well-being in old age: Do macro-contexts make a difference». International Congress Series, vol. 1282, 147-151.

HIRVENSALO, M., LAMPINEN, P. y RANTANEN, T. (1998): «Physical exercise in old age: An eight-year follow-up study on involvement, motives, and obstacles among persons aged 65-84». Journal of Aging and Physical Activity, vol. 6, nº 2, 157-168.

HOOYMAN, N. y KIYAK, H.A. (1996): Social Gerontology: A Multidisciplinary Perspectiva, $4^{\mathrm{a}}$ ed. Boston. Allyn \& Bacon.

INE (2007): Encuesta sobre equipamiento y uso de tecnologías de la información y comunicación en los hogares. INEBASE. Madrid. Instituto Nacional de Estadístca.

INSTITUTO DE MAYORES Y SERVICIOS SOCIALES (2004): Encuesta de condiciones de vida de los mayores, Estudio 4.597. Madrid. Instituto de Mayores y Servicios Sociales. Disponible en http://www.imsersomayores.csic.es/estadisticas/encuestas/index.html.

INSTITUTO DE ESTADÍSTICA DE LA COMUNIDAD DE MADRID (2001): Zonificación estadística NUTS 4 de la Comunidad de Madrid. Madrid. Instituto de 
Estadística, Comunidad de Madrid. Disponible en http://www.madrid.org/iestadis/fijas clasificaciones/conuts4.htm.

INSTITUTO DE ESTADÍSTICA DE LA COMUNIDAD DE MADRID. (2005). Padrón Continuo de Habitantes, actualizado a enero de 2005. Instituto de Estadística, Comunidad de Madrid.

IWASAKI, Y. (2007): «Leisure and Quality of Life in and International and Multicultural Context: What are major Pathways linking Leisure to Quality of Life?». Social Indicators Research, vol. 82, n 2, 233-264.

JANKE, M.C., PAYNE, L.L. y PUYMBROECK, M.V. (2008): «The Role of Informal and Formal Leisure Activities in the Disablement Process». The International Journal of Aging and Human Development, vol. 67, n 3, 231-257.

JONES, M. (1990): «Time use of the elderly». Canadian Social Trends, vol. 17, 28-30.

KING, A., TAYLOR, C. y HESKILL, W. (1993): «Effects of differing intensities and formats of 12 months of exercise training on psychological outcomes in older adults». Health Psychology, vol. 12, n 4, 292-300.

KUBECK, J.E., SALLY, A.M.-A. y MARTIN, D.M. (1999): «Finding Information on the Web: Exploring Older Adults Exploration». Educational Gerontology, vol. 25, nº 2, 167-183.

LAN, T.-Y., CHANG, H.-Y. y TAI, T.-Y. (2006): «Relationship between components of leisure physical activity and mortality in Taiwanese older adults». Preventive Medicine, vol. $43, n^{\circ} 1,36-41$.

LASSEY, W.R. y LASSEY, M.L. (2001): Quality of Life for Older People. An International Perspective. New Jersey. Prentice Hall.

LEFRANÇOIS, R., LECLERC, G. y POULIN, N. (1997): «Predictors of Activity Involvement among Older Adults». Activities, Adaptation \& Aging, vol. 22, n 4, 15-29.

LLOYD, K.M. y AULD, C.J. (2002): «The Role of Leisure in Determining Quality of Life: Issues of Content and Measurement». Social Indicators Research, vol. 57, nº 1, 43-71.

LU, L., y GILMOUR, R. (2004): «Culture and conceptions of happiness: Individual oriented and social oriented». Journal of Happiness Studies, vol. 5, n 3, 269-291.

LU, L. y HU, C.-H. (2005): «Personality, leisure experiences and happiness». Journal of Happiness Studies, vol. 6, n $3,325-342$.

MALMBERG, J., MIILUNPALO, S., PASANEN, M., VUORI, I. y OJA, P. (2005): «Characteristics of leisure time physical activity associated with risk of decline in perceived health -a 10-year follow-up of middle-aged and elderly men and women». Preventive Medicine, vol. 41, $\mathrm{n}^{\circ}$ 1, 141-150.

MARTÍNEZ-MARTÍN, P., FERNÁNDEZ-MAYORALAS, G., FRADES-PAYO, B., ROJOPÉREZ, F., RODRÍGUEZ-RODRÍGUEZ, V., FORJAZ, M.J., PRIETO-FLORES, M.E. y DE PEDRO CUESTA, J. (2009): «Validación de la Escala de Independencia Funcional». Gaceta Sanitaria, vol. 23, n 1, 49-54.

McPHERSON, B.D. y YAMAGUCHI, Y. (1995): «Aging and active lifestyles: A cross-cultural analysis of factors influencing the participation of middle-aged and elderly cohorts» en Physical Activity, Aging and Sports, vol. IV (Harris, S., Heikkinen, E. y Harris, W. S., eds.). Albany, NY, Edit. Center for the Study of Aging, 293-308.

MENEC, V.H. y CHIPPERFIELD, J.G. (1997): «Remaining active in later life, the role of locus of control in seniors' leisure activity participation, health and life satisfaction». Journal of Aging and Health, vol. 9, $\mathrm{n}^{\circ}$ 1, 105-125. 
MERCER, C. (1994): «Assessing liveability: from statistical indicators to policy benchmarks» en Urban and Regional Quality of Life Indicators (Mercer, C., ed.). Brisbane, Edit. Institute for Cultural Policy Studies, Griffith University, 3-12.

MOLLER, V. (1992): «Spare time use and perceived well-being among black South African youth». Social Indicators Research, vol. 26, no 4, 309-351.

MORAGAS, R. (2001): La jubilación. Una oportunidad vital. Barcelona. Herder.

NEAL, J.D., SIRGY, M.J. y UYSAL, M. (1999): «The role of satisfaction with leisure travel/ tourism services en experiences in satisfaction with leisure life and overall life». Journal of Business Research, vol. 44, n 3, 153-163.

NILSSON, I., LÖFGREN, B., FISHER, A.G. y BERNSPANG, B. (2006): «Focus on Leisure Repertoire in the Oldest Old: The Umea 85+ Study». The Journal of Applied Gerontology, vol. 25, $\mathrm{n}^{\circ} 5,391-405$.

NIMROD, G. (2007): «Retirees`Leisure: Activities, Benefits, and their Contribution to Life Satisfaction». Leisure Studies, vol. 26, nº 1, 65-80.

NIMROD, G. (2008): «In support of innovation theory: innovation in activity patterns and life satisfaction among recently retired individuals». Ageing \& Society, vol. 28, $\mathrm{n}^{\circ}$ 6, 831846.

NORUSIS, M.J. (1990): SPSS/PC+ Statistics 4.0 for the IBM PC/XT/AT and SP/2. SPSS Inc., s.p., Chicago.

O'BRIEN COUSINS, S. (1995): «The life situational determinants of exercise in women over age 70» en Physical Activity, Aging and Sports, vol. IV (Harris, S., Heikkinen, E. y Harris, W. S., eds.). Albany, NY, Edit. Center for the Study of Aging, 259-277.

OECD (2000): Reforms for an Aging Society. Paris. Organisation for Economic Co-operation and Development.

ONISHI, J., MASUDA, Y., SUZUKI, Y., GOTOH, T., KAWAMURA, T. y IGUCHI, A. (2005): «The pleasurable recreational activities among community-dwelling older adults». Archives of Gerontology and Geriatrics, vol. 43, n 2, 147-155.

OVERS, R.P., TAYLOR, S., CASSELL, E. y CHERNOV, M. (1977): Avocational counseling for the elderly. Sussex. Avocational Counseling Research.

PAILLARD-BORG, S., WANG, H.-X., WINBLAD, B. y FRATIGLIONI, L. (2009): «Pattern of participation in leisure activities among older people in relation to their health conditions and contextual factors: a survey in a Swedish urban area». Ageing \& Society, vol. $29, \mathrm{n}^{\circ} 5,803-821$.

PATTERSON, I. (1996): «Participation in leisure activities by older adults after a stressful life event: the loss of a spouse». Internacional Journal of Aging and Human Development, vol. 42, no 2, 123-142.

PRIETO-FLORES, M.E., FERNÁNDEZ-MAYORALAS, G., ROJO-PÉREZ, F., LARDIÉSBOSQUE, R., RODRÍGUEZ-RODRÍGUEZ, V., AHMED-MOHAMED, K. y ROJOABUÍN, J.M. (2008): «Factores sociodemográficos y de salud en el bienestar emocional como dominio de calidad de vida de las personas mayores en la Comunidad de Madrid, 2005». Revista Española de Salud Pública, vol. 82, n 3, 301-313.

RAKOWSKI, W. y MOR, V. (1992): «The association of physical activity with mortality among older adults in the longitudinal study of aging (1984-1988)». The Journals of Gerontology, vol. 47, nº 4, 122-129. 
RODRÍGUEZ-RODRÍGUEZ, V., ROJO-PÉREZ, F., FERNÁNDEZ-MAYORALAS, G., AHMED-MOHAMED, K., LARDIÉS-BOSQUE, R., PRIETO-FLORES, M.E. y ROJOABUÍN, J.M. (2011): «Recursos económicos y calidad de vida en la población mayor». Revista Internacional de Sociología, vol. 69, nº 1, 195-227.

ROJO-PÉREZ, F., FERNÁNDEZ-MAYORALAS, G., RODRÍGUEZ-RODRÍGUEZ, V. y ROJO-ABUÍN, J. M. (2007): «The environments of ageing in the context of the global Quality of Life among the elderly living in family housing» en Quality of Life in Old Age. International and Multi-disciplinary Perspectives (Mollenkopf, H. y Walker, A., eds.). Dordrecht, The Netherlands, Edit. Springer, Social Indicators Research Series, núm. 31, 123-150.

ROJO-PÉREZ, F., FERNÁNDEZ-MAYORALAS, G., AHMED-MOHAMED, K., PRIETOFLORES, M ${ }^{\mathrm{a}}$.E., ROJO-ABUÍN, J.M., MARTÍNEZ-MARTÍN, P., FORJAZ, M. J. y DELGADO-SANZ, M.C. (2008): Población mayor, Calidad de Vida y redes de apoyo: demanda y prestación de cuidados en el seno familiar, $2^{\circ}$ Premio, Edición 2008. Madrid. Caja Madrid de Investigación Social.

SCHOOLER, C. y MULATU, M.S. (2001): «The Reciprocal Effects of Leisure Time Activities and Intellectual Functioning in Older People: A Longitudinal Analysis». Psychology and Aging, vol. 16, $\mathrm{n}^{\mathrm{o}} 3$ 3, 466-482.

SCOTT, D. y WILLITS, F.K. (1998): «Adolescent and adult leisure patterns: A Reassessment». Journal of Leisure Research, vol. 30, n 3, 319-330.

SEEMAN, T.E., BERKMAN, L.F., CHARPENTIER, P.A., BLAZER, D.G., ALBERT, M.S. y TINETTI, M.E. (1995): «Behavioral and Psychosocial Predictors of Physical Performance: MacArthur Studies of Successful Aging». Journal of Gerontology: Medical Sciences, vol. 50, n $4,177-183$.

SILVERSTEIN, M. y PARKER, M.G. (2002): «Leisure Activities and Quality of Life among the Oldest Old in Sweden». Research on Aging, vol. 24, nº 5, 528-547.

SMITH, A. (2000): Researching quality of life of older people: concepts, measures and findings. WP, $\mathrm{n}^{\mathrm{o}}$ 7, Keele University. Staffordshire, UK, Centre for Social Gerontology. Disponible en Www.keele.ac.uk/csg/.../research_quality.pdf.

SOTGIU, I., GALATO, D. y MANZANO, M. (2011): «Happiness Components and their Attainment in Old Age: A Cross-Cultural Comparison Between Italy and Cuba». Journal of Happiness Studies, vol. 12, no 3, 353-371.

THE EUROQoL GROUP (1990): «Euroqol - a new facility for the measurement of health related quality of life». Health Policy, vol. 16, n 3, 199-208.

TOMLINSON, M. (2003): «Lifestyle and Social Class». European Sociological Review, vol. $19, \mathrm{n}^{\circ} 1,97-111$.

WADE, D.T. y COLLIN, C. (1988): «The Barthel ADL Index: a standard measure of physical disability?». International Disability Studies, vol. 10, $\mathrm{n}^{\circ} 2,64-67$.

WANG, H.-X., KARP, A., WINBLAD, B. y FRATIGLIONI, L. (2002): «Late-life engagement in social and leisure activities is associated with a decreased risk of dementia: a longitudinal study from the Kungsholmen Project». American Journal of Epidemiology, vol. $155, \mathrm{n}^{\mathrm{o}} 12,1081-1087$.

ZIGMOND, A.S. y SNAITH, R.P. (1983): «The hospital anxiety and depression scale». Acta Psychiatrica Scandinavica, vol. 67, nº 6, 361-370. 
